\title{
SOLUÇÕES DA EQUAÇÃO DA CONDUÇÃO DO CALOR BIDIMENSIONAL COM CONDUTIVIDADE TÉRMICA DEPENDENTE DA TEMPERATURA E GERAÇÃO DE CALOR
}

\author{
E. T. CABRAL ${ }^{1}$, F. A. PONTES ${ }^{1}$, H. K. MIYAGAWA ${ }^{2}$, E. N. MACÊDO ${ }^{3}$ e J. N. N. QUARESMA ${ }^{3}$ \\ ${ }^{1}$ Universidade Federal do Pará, Programa de Pós-Graduação em Engenharia Química \\ ${ }^{2}$ Universidade Federal do Pará, Programa de Pós-Graduação em Engenharia de Recursos Naturais da \\ Amazônia \\ ${ }^{3}$ Universidade Federal do Pará, Faculdade de Engenharia Química \\ E-mail para contato: et.cabral@yahoo.com.br
}

\begin{abstract}
RESUMO - O presente trabalho busca aplicar a solução híbrida da Técnica da Transformada Integral Generalizada na solução da equação da condução de calor em aletas longitudinais bidimensionais de perfil não-variável com condutividade térmica dependente da temperatura com geração de energia decaindo exponencialmente com a posição caracterizando um problema não-linear e não-homogêneo. Para isso, foi utilizada a formulação em termos de variáveis adimensionais com objetivo de simplificar o estudo do domínio da solução, a partir do balanço de energia aplicado no volume de controle. Foram estudados dois casos limites para Biot tendendo a zero e infinito obtendo-se resultados semelhantes aos obtidos pela sub-rotina $F P S 2 H$ da biblioteca ISML contida na linguagem de programação científica Fortran 90/95.
\end{abstract}

\section{INTRODUÇÃO}

Uma variedade de problemas de condução de calor não-lineares tem sido tratada pela Técnica da Transformada Integral Generalizada (em inglês GITT), a qual tem se mostrado como uma poderosa ferramenta na solução dessa classe de problemas (Cotta, 1990; Cotta, 1993; Cotta e Mikhailov, 1997). Essa técnica é baseada no uso de expansões de autofunções ortogonais para expressar as variáveis dependentes desconhecidas (Silva e Sphaier, 2010; Sphaier et al., 2011).

Aletas (ou superfícies estendidas) são projetadas com o objetivo de intensificar a transferência de calor através do aumento da área de troca térmica em equipamentos térmicos (Ramos, 1993), em função disso sua análise desempenha um papel fundamental no estabelecimento da eficiência da transferência de calor para diversas geometrias (Malik e Rafiq, 2010).

O presente trabalho visa contribuir com o entendimento do fenômeno de transferência de calor em superfícies estendidas de perfil não-variável a partir da aplicação da GITT em um problema tido como complexo em virtude da condutividade térmica ser dependente da temperatura e a existência do termo de geração de energia, o qual decai exponencialmente com a posição caracterizando, desta 
forma, um problema não-linear e não-homogêneo. A Figura 1 a seguir apresenta o modelo físico conceitual para o problema a ser estudado.

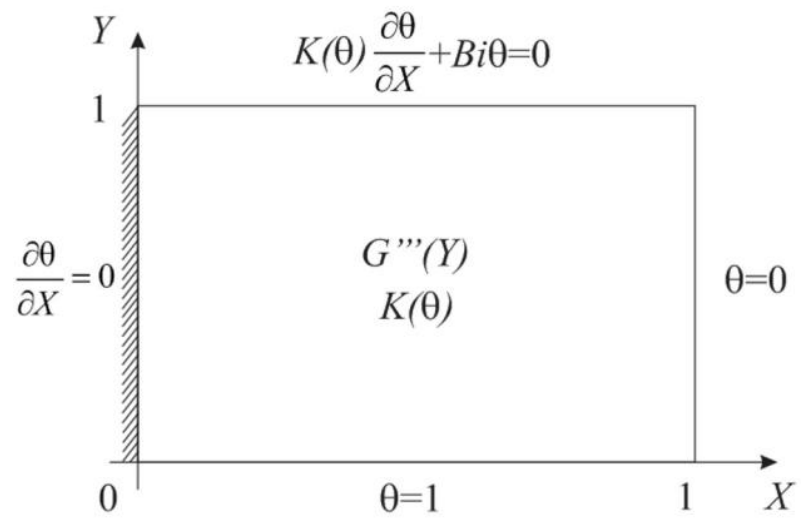

Figura 1 - Representação conceitual do problema estudado.

\section{ANÁLISE}

O problema da condução de calor bidimensional com condutividade térmica variável e geração de calor na sua forma adimensional pode ser escrito como:

$$
\begin{array}{ll}
\frac{\partial}{\partial X}\left[K(\theta) \frac{\partial \theta}{\partial X}\right]+\gamma^{2} \frac{\partial}{\partial Y}\left[K(\theta) \frac{\partial \theta}{\partial Y}\right]=-G_{0} e^{-\eta Y}, & 0<X<1,0<Y<1 \\
\frac{\partial \theta(0, Y)}{\partial X}=0 ; \quad \theta(1, Y)=0, & 0 \leq Y \leq 1 \\
\theta(X, 0)=1 ; \quad K(\theta) \frac{\partial \theta(X, 1)}{\partial Y}+\operatorname{Bi} \theta(X, 1)=0, & 0<X<1
\end{array}
$$

Com os seguintes grupos adimensionais:

$$
\begin{aligned}
& \theta=\frac{T-T_{\infty}}{T_{w}-T_{\infty}} ; X=\frac{x}{L_{x}} ; Y=\frac{y}{L_{y}} ; \mathrm{K}(\theta)=\frac{k}{k_{0}}=1+b \theta ; b=\frac{k_{1}\left(T_{w}-T_{\infty}\right)}{k_{0}} ; \\
& B i=\frac{h L_{y}}{k_{0}} ; \eta=c L_{y} ; \gamma=\frac{L_{x}}{L_{y}} ; G_{0}=\frac{g_{0} L_{x}^{2}}{k_{0}\left(T_{w}-T_{\infty}\right)}
\end{aligned}
$$

Propondo-se $K(\theta)=U(X, Y)=1+b \theta(X, Y)$ e $W(X, Y)=U^{2}(X, Y)$, as Equações 1 a 3 são modificadas, resultando: 
$\frac{\partial^{2} W}{\partial X^{2}}+\gamma^{2} \frac{\partial^{2} W}{\partial Y^{2}}=-G^{*}(Y)$

$\frac{\partial W(0, Y)}{\partial X}=0 ; \quad W(1, Y)=1, \quad 0 \leq Y \leq 1$

$W(X, 0)=d^{2}=f ; \quad \frac{\partial W(X, 1)}{\partial Y}+B i^{*} W(X, 1)=B i_{m}, \quad 0<X<1$

em que:

$$
\begin{aligned}
& G^{*}(Y)=2 b G^{\prime \prime \prime}(Y)=2 b G_{0} e^{-\eta Y} \\
& B i^{*}=\frac{2 B i}{W^{1 / 2}}=\frac{B i_{m}}{W^{1 / 2}}
\end{aligned}
$$

O sistema composto pelas Equações 6, 7 e 8 é simplificado em um problema particular e um filtrado ( $W_{P}$ e $W_{F}$, respectivamente) a partir da seguinte proposta de solução:

$$
W(X, Y)=W_{P}(X, Y)+W_{F}(X, Y)
$$

\subsection{Problema particular - $W_{P}(X, Y)$}

$$
\begin{aligned}
& \frac{\partial^{2} W_{P}}{\partial X^{2}}+\gamma^{2} \frac{\partial^{2} W_{P}}{\partial Y^{2}}=G^{*}(Y) \\
& \frac{\partial W_{P}(0, Y)}{\partial X}=0 ; \quad W_{P}(1, Y)=1, \quad 0 \leq Y \leq 1 \\
& W(X, 0)=f ; \quad \frac{\partial W_{P}(X, 1)}{\partial Y}+B i_{m} W_{P}(X, 1)=B i_{m}, \quad 0<X<1
\end{aligned}
$$

Considerando-se a seguinte proposta de solução:

$W_{P}(X, Y)=W_{P 1}(Y)+W_{P 2}(X, Y)$

Problema particular $-W_{P 1}(Y)$ :

$$
\frac{d^{2} W_{P 1}}{d Y^{2}}+2 b G_{0} e^{-\eta Y}=0
$$


$W_{P 1}(0)=f ; \quad \frac{d W_{P 1}(1)}{d X}+B i_{m} W_{P 1}(1)=B i_{m}$

Problema filtrado $-W_{P 2}(X, Y)$ :

$$
\begin{array}{ll}
\frac{\partial^{2} W_{P 2}}{\partial X^{2}}+\gamma^{2} \frac{\partial^{2} W_{P 2}}{\partial Y^{2}}=0 & \\
\frac{\partial W_{P 2}(0, Y)}{\partial X}=0 ; \quad W_{P 2}(1, Y)=1-W_{P 1}, & 0 \leq Y \leq 1 \\
W_{P 2}(X, 0)=0, \quad \frac{\partial W_{P 2}(X, 1)}{\partial Y}+B i_{m} W_{P 2}(X, 1)=0, & 0<X<1
\end{array}
$$

O sistema formado pelas Equações 16 e 17 é solucionado analiticamente. O descrito pelas Equações 18,19 e 20 possui solução analítica pela Técnica da Transformada Integral Clássica (em inglês CITT), cujo problema de autovalor associado é:

$$
\begin{aligned}
& \frac{d^{2} \Gamma_{l}}{d Y^{2}}+\beta_{l}^{2} \Gamma_{l}=0 \\
& \Gamma_{l}(0)=0 ; \quad \frac{d \Gamma_{l}(1)}{d Y}+B i_{m} \Gamma_{l}(1)=0
\end{aligned}
$$

onde os autovalores são as raízes da equação:

$\beta_{l} \cos \left(\beta_{l}\right)+B i_{m} \sin \left(\beta_{l}\right)=0$

e a autofunção é:

$$
\Gamma_{l}(Y)=\operatorname{sen}\left(\beta_{l} Y\right)
$$

A norma é definida por:

$$
N_{l}=\int_{0}^{1} \Gamma_{l}^{2}(Y) d Y=\int_{0}^{1}\left[\operatorname{sen}\left(\beta_{l} Y\right)\right]^{2} d Y=\frac{1}{2}-\frac{\operatorname{sen}\left(2 \beta_{l}\right)}{4 \beta_{l}}
$$

O par transformada-inversa é:

$$
\bar{W}_{P 2 l}(X)=\int_{0}^{1} \tilde{\Gamma}_{l}(Y) W_{P 2}(X, Y) d Y \quad \text { Transformada }
$$


$W_{P 2}(X, Y)=\sum_{l=1}^{\infty} \tilde{\Gamma}_{l}(Y) \bar{W}_{P 2 l}(X) \quad$ Inversa

em que a autofunção normalizada é:

$\tilde{\Gamma}_{l}=\frac{\Gamma_{l}}{\sqrt{N_{l}}}$

Operando-se o sistema de Equações 18, 19 e 20 com a integral $\int_{0}^{1} \tilde{\Gamma}_{l}(Y) \_d Y$, resulta:

$\frac{d^{2} \bar{W}_{P 2 l}}{d X^{2}}-\beta_{l}^{2} \gamma^{2} \bar{W}_{P 2 l}=0$
$\frac{d \bar{W}_{P 2 l}(0)}{d X}=0 ; \quad \bar{W}_{P 2 l}(1)=\int_{0}^{1} \tilde{\Gamma}_{l}\left(1-W_{P 1}\right) d Y$

O sistema de Equações 29 e 30 foi solucionado analiticamente para cada índice $l$ de forma analítica utilizando a rotina DSolve do software Mathematica.

\subsection{Problema filtrado - $W_{F}(X, Y)$}

$$
\begin{aligned}
& \frac{\partial^{2} W_{F}}{\partial X^{2}}+\gamma^{2} \frac{\partial^{2} W_{F}}{\partial Y^{2}}=0 \\
& \frac{\partial W_{F}(0, Y)}{\partial X}=0 ; \quad W_{F}(1, Y)=0, \quad 0 \leq Y \leq 1 \\
& W_{F}(X, 0)=\left(f-W_{P}\right) ; \\
& \frac{\partial W_{F}(X, 1)}{\partial Y}+\frac{\partial W_{P}(X, 1)}{\partial Y}+B i_{m}\left(W_{P}(X, 1)+W_{F}(X, 1)\right)^{1 / 2}=B i_{m}, \quad 0<X<1
\end{aligned}
$$

O sistema de Equações 31, 32 e 33 é solucionado pela GITT. Cujo problema de autovalor associado é definido pelas Equações 34 e 35 a seguir:

$$
\begin{aligned}
& \frac{d^{2} \Psi_{i}}{d X^{2}}+\mu_{i}^{2} \Psi_{i}=0 \\
& \frac{d \Psi_{i}(0)}{d X}=0 ; \quad \Psi_{i}(1)=0
\end{aligned}
$$


onde os autovalores $\mu_{i}$ são calculados a partir de:

$$
\cos \left(\mu_{i}\right)=0
$$

e a autofunção é definida como:

$$
\Psi_{i}(X)=\cos \left(\mu_{i} X\right)
$$

A norma é calculada:

$$
N_{i}=\int_{0}^{1} \Psi_{i}^{2}(X) d X=\int_{0}^{1}\left[\cos \left(\mu_{i} X\right)\right]^{2} d X=\frac{1}{2}
$$

O par transformada-inversa para $W_{F}(X, Y)$ é:

$\bar{W}_{F i}(Y)=\int_{0}^{1} \tilde{\Psi}_{i}(X) W_{F}(X, Y) d X \quad$ Transformada

$W_{F}(X, Y)=\sum_{i=1}^{\infty} \tilde{\Psi}_{i}(X) \bar{W}_{F i}(Y) \quad$ Inversa

onde a autofunção normalizada é:

$$
\tilde{\Psi}_{i}=\frac{\Psi_{i}}{\sqrt{N_{i}}}
$$

Operando-se as Equações 18, 19 e 20 com a integral $\int_{0}^{1} \tilde{\Psi}_{i}(X) \_d X$, o resultado é:

$$
\begin{aligned}
& \frac{d^{2} \bar{W}_{F i}}{d Y^{2}}-\frac{\mu_{i}^{2}}{\gamma^{2}} \bar{W}_{F i}=0 \\
& \bar{W}_{F i}=\int_{0}^{1} \tilde{\Psi}_{i}\left(f-W_{P}\right) d X ; \quad \sum_{j=0}^{n t} \sum_{k=0}^{n t} C_{1 i j k} \bar{W}_{F j} \bar{W}_{F k}+\sum_{j=0}^{n t} C_{3 i j} \bar{W}_{F j}-2 B i_{m} \frac{d \bar{W}_{F i}}{d Y}-B i_{m}{ }^{2} \bar{W}_{F i}=C_{2 i}
\end{aligned}
$$

O sistema definido pelas Equações 42 e 43 foi solucionado numericamente pela rotina NDSolve do software Mathematica, onde os coeficientes são calculados pelas Equações 44 a seguir:

$$
C_{1 i j k}=\int_{0}^{1} \tilde{\Psi}_{i} \tilde{\Psi}_{j} \tilde{\Psi}_{k} d X ; \quad C_{2 i}=\int_{0}^{1} \tilde{\Psi}_{i} W_{P} d X ; \quad C_{3 i j}=2 \int_{0}^{1} \tilde{\Psi}_{i} \tilde{\Psi}_{j} \frac{\partial W_{P}}{\partial Y} d X
$$




\section{RESULTADOS E DISCUSSÃO}

Dois casos limites foram escolhidos para verificação da solução proposta neste trabalho, e os resultados foram comparados com os obtidos através método de diferenças finitas pela subrotina FPS2H da biblioteca IMSL (IMSL, 1991) contida na linguagem de programação Fortran 90/95. No Caso 1 considera-se $b=0, B i \rightarrow \infty$ e as demais constantes iguais à unidade enquanto que no Caso $2, b=O, B i \rightarrow O$ e as demais constantes iguais à unidade.

A convergência da solução pela GITT foi realizada considerando o número de termos do problema particular $(N T P)$ e o número de termos do problema filtrado $(N T F)$. A Tabela 1 mostra a convergência para os Casos 1 e 2 para $N T P=35$ e $N T F=0,10$ e 15 .

Tabela 1 - NTP $=35$, NTF variável

\begin{tabular}{|c|c|c|c|c|c|c|c|c|c|}
\hline \multicolumn{5}{|c|}{ Caso 1} & \multicolumn{5}{|c|}{ Caso 2} \\
\hline \multicolumn{5}{|c|}{$N T F=0$} & \multicolumn{5}{|c|}{$N T F=0$} \\
\hline$X \backslash Y$ & 0.0 & 0.3 & 0.6 & 1.0 & $X \backslash Y$ & 0.0 & 0.3 & 0.6 & 1.0 \\
\hline 0.0 & 1.00000 & 0.85955 & 0.72701 & 0.65252 & 0.0 & 1.00000 & 0.71837 & 0.41384 & 0.00000 \\
\hline 0.5 & 1.00000 & 0.73097 & 0.55824 & 0.48166 & 0.5 & 1.00000 & 0.63035 & 0.33345 & 0.00000 \\
\hline 1.0 & 1.00000 & 0.00056 & -0.01124 & -0.00909 & 1.0 & 1.00000 & -0.00847 & -0.00660 & 0.00000 \\
\hline \multicolumn{5}{|c|}{$N T F=10$} & \multicolumn{5}{|c|}{$N T F=10$} \\
\hline$X \backslash Y$ & 0.0 & 0.3 & 0.6 & 1.0 & $X \backslash Y$ & 0.0 & 0.3 & 0.6 & 1.0 \\
\hline 0.0 & 1.00000 & 0.85955 & 0.72701 & 0.65252 & 0.0 & 1.00000 & 0.71837 & 0.41384 & 0.00000 \\
\hline 0.5 & 1.00000 & 0.73097 & 0.55824 & 0.48166 & 0.5 & 1.00000 & 0.63035 & 0.33345 & 0.00000 \\
\hline 1.0 & 1.00000 & 0.00056 & -0.01124 & -0.00909 & 1.0 & 1.00000 & -0.00847 & -0.00660 & 0.00000 \\
\hline \multicolumn{5}{|c|}{$N T F=15$} & \multicolumn{5}{|c|}{$N T F=15$} \\
\hline $\mathrm{X} \backslash \mathrm{Y}$ & 0.0 & 0.3 & 0.6 & 1.0 & $X \backslash Y$ & 0.0 & 0.3 & 0.6 & 1.0 \\
\hline 0.0 & 1.00000 & 0.85955 & 0.7270 & 0.65252 & 0.0 & 1.00000 & 0.71837 & 0.41384 & 0.00000 \\
\hline 0.5 & 1.00000 & 0.73097 & 0.55824 & 0.48166 & 0.5 & 1.00000 & 0.63035 & 0.33345 & 0.00000 \\
\hline 1.0 & 1.00000 & 0.00056 & -0.01124 & -0.00909 & 1.0 & 1.00000 & -0.00847 & -0.00660 & 0.00000 \\
\hline
\end{tabular}

Como pode ser notado na Tabela 1 , nos casos estudados, o problema não é influenciado pelo número de termos na solução do problema filtrado. Já a Tabela 2 mostra a convergência da solução para $N T F=10$ com $N T P=10,30$ e 40. Nota-se a convergência da solução de acordo com o aumento do número de termos da solução particular e a dificuldade da convergência em $X$ $=1$. 
Tabela 2 - NTF = 10, NTP variável

\begin{tabular}{|c|c|c|c|c|c|c|c|c|c|}
\hline \multicolumn{5}{|c|}{ Caso 1} & \multicolumn{5}{|c|}{ Caso 2} \\
\hline \multicolumn{5}{|c|}{$N T P=10$} & \multicolumn{5}{|c|}{$N T P=10$} \\
\hline $\mathrm{X} \mid \mathrm{Y}$ & 0.0 & 0.3 & 0.6 & 1.0 & $\mathrm{X} \backslash \mathrm{Y}$ & 0.0 & 0.3 & 0.6 & 1.0 \\
\hline 0.0 & 1.00000 & 0.85955 & 0.72701 & 0.65252 & 0.0 & 1.00000 & 0.71837 & 0.41384 & 0.00000 \\
\hline 0.4 & 1.00000 & 0.78208 & 0.62097 & 0.54315 & 0.4 & 1.00000 & 0.66724 & 0.36501 & 0.00000 \\
\hline 0.9 & 1.00000 & 0.22765 & 0.14152 & 0.11551 & 0.9 & 1.00000 & 0.20554 & 0.09029 & 0.00000 \\
\hline 1.0 & 1.00000 & -0.06881 & 0.03919 & 0.03178 & 1.0 & 1.00000 & -0.06118 & 0.02299 & 0.00000 \\
\hline \multicolumn{5}{|c|}{$N T P=30$} & \multicolumn{5}{|c|}{$N T P=30$} \\
\hline $\mathrm{X} \backslash \mathrm{Y}$ & 0.0 & 0.3 & 0.6 & 1.0 & $\mathrm{X} \backslash \mathrm{Y}$ & 0.0 & 0.3 & 0.6 & 1.0 \\
\hline 0.0 & 1.00000 & 0.85955 & 0.72701 & 0.65252 & 0.0 & 1.00000 & 0.71837 & 0.41384 & 0.00000 \\
\hline 0.4 & 1.00000 & 0.78208 & 0.62097 & 0.54315 & 0.4 & 1.00000 & 0.66724 & 0.36501 & 0.00000 \\
\hline 0.9 & 1.00000 & 0.23020 & 0.13989 & 0.11417 & 0.9 & 1.00000 & 0.20777 & 0.08935 & 0.00000 \\
\hline 1.0 & 1.00000 & -0.02332 & 0.01311 & 0.01061 & 1.0 & 1.00000 & -0.02077 & 0.00770 & 0.00000 \\
\hline \multicolumn{5}{|c|}{$N T P=40$} & \multicolumn{5}{|c|}{$N T P=40$} \\
\hline $\mathrm{X} \backslash \mathrm{Y}$ & 0.0 & 0.3 & 0.6 & 1.0 & $\mathrm{X} \backslash \mathrm{Y}$ & 0.0 & 0.3 & 0.6 & 1.0 \\
\hline 0.0 & 1.00000 & 0.85955 & 0.72701 & 0.65252 & 0.0 & 1.00000 & 0.71837 & 0.41384 & 0.00000 \\
\hline 0.4 & 1.00000 & 0.78208 & 0.62097 & 0.54315 & 0.4 & 1.00000 & 0.66724 & 0.36501 & 0.00000 \\
\hline 0.9 & 1.00000 & 0.23020 & 0.13989 & 0.11417 & 0.9 & 1.00000 & 0.20777 & 0.08935 & 0.00000 \\
\hline 1.0 & 1.00000 & 0.01751 & 0.00983 & 0.00796 & 1.0 & 1.00000 & 0.01560 & 0.00578 & 0.00000 \\
\hline
\end{tabular}

Nas Tabelas 3 e 4 os dados dos Casos 1 e 2, com 40 termos, podem ser observados e comparados com a solução do mesmo problema pela sub-rotina FPS2H. A sub-rotina converge para uma malha uniforme 101x101.

Tabela 3 - Resultados Caso 1

\begin{tabular}{ccccccc}
\hline \multicolumn{7}{c}{ GITT (Presente Trabalho) } \\
\hline $\mathrm{X} \backslash \mathrm{Y}$ & 0.0 & 0.2 & 0.4 & 0.6 & 0.8 & 1.0 \\
\hline 0.0 & 1.00000 & 0.90960 & 0.81081 & 0.72701 & 0.67187 & 0.65252 \\
0.4 & 1.00000 & 0.85293 & 0.71881 & 0.62097 & 0.56269 & 0.54315 \\
0.8 & 1.00000 & 0.53476 & 0.34727 & 0.26870 & 0.23264 & 0.22161 \\
0.9 & 1.00000 & 0.31545 & 0.18501 & 0.13989 & 0.12017 & 0.11417 \\
1.0 & - & 0.02567 & 0.01353 & 0.00983 & 0.00837 & 0.00796 \\
\hline \multicolumn{7}{c}{ FPS2H } \\
\hline $\mathrm{X} \backslash \mathrm{Y}$ & 0.0 & 0.2 & 0.4 & 0.6 & 0.8 & 1.0 \\
\hline 0.0 & 1.00000 & 0.90960 & 0.81081 & 0.72701 & 0.67187 & 0.65252 \\
0.4 & 1.00000 & 0.85293 & 0.71881 & 0.62097 & 0.56269 & 0.54315 \\
0.8 & 1.00000 & 0.53476 & 0.34727 & 0.26870 & 0.23264 & 0.22161 \\
0.9 & 1.00000 & 0.31545 & 0.18501 & 0.13989 & 0.12017 & 0.11417 \\
1.0 & - & 0.00000 & 0.00000 & 0.00000 & 0.00000 & 0.00000 \\
\hline
\end{tabular}


Na Tabela 3 nota-se que os resultados obtidos pela GITT possuem boa concordância com os obtidos pela sub-rotina $F P S 2 H$ principalmente para pontos não muito próximos do contorno em $X=1$. Na Tabela 4, para o Caso 2, o comportamento observado na Tabela 3 é repetido.

Tabela 4: Resultados Caso 2

\begin{tabular}{ccccccc}
\hline \multicolumn{7}{c}{ GITT (Presente Trabalho) } \\
\hline $\mathrm{X} \backslash \mathrm{Y}$ & 0.0 & 0.2 & 0.4 & 0.6 & 0.8 & 1.0 \\
\hline 0.0 & 1.00000 & 0.81732 & 0.61734 & 0.41384 & 0.20975 & 0.00000 \\
0.4 & 1.00000 & 0.77793 & 0.56127 & 0.36501 & 0.18242 & 0.00000 \\
0.8 & 1.00000 & 0.50589 & 0.28642 & 0.16912 & 0.08244 & 0.00000 \\
0.9 & 1.00000 & 0.30082 & 0.15417 & 0.08935 & 0.04372 & 0.00000 \\
1.0 & - & 0.02441 & 0.01094 & 0.00578 & 0.00259 & 0.00000 \\
\hline \multicolumn{7}{c}{ FPS2H } \\
\hline $\mathrm{X} \backslash \mathrm{Y}$ & 0.0 & 0.2 & 0.4 & 0.6 \\
\hline 0.0 & 1.00000 & 0.81732 & 0.61734 & 0.41384 & 0.20975 & 0.00000 \\
0.4 & 1.00000 & 0.77793 & 0.56127 & 0.36501 & 0.18242 & 0.00000 \\
0.8 & 1.00000 & 0.50589 & 0.28642 & 0.16912 & 0.08244 & 0.00000 \\
0.9 & 1.00000 & 0.30082 & 0.15417 & 0.08935 & 0.04372 & 0.00000 \\
1.0 & - & 0.00000 & 0.00000 & 0.00000 & 0.00000 & 0.00000 \\
\hline
\end{tabular}

Em ambos os casos estudados, os valores em $X=1$ apresentam comportamento oscilatório característicos de soluções por séries. Porém, esse comportamento é suavizado aumentando-se o número de termos na solução do problema particular. Além disso, é observado nas Tabelas 3 e 4 que em $X=1$ esse comportamento também é suavizado nos pontos próximos de $Y=1$.

Verifica-se que nas Tabelas 3 e 4 no ponto $(1,0)$ para os resultados da GITT e da sub-rotina $F P S 2 H$ não são informados valores em virtude de nesses pontos ocorrerem singularidades.

\section{CONCLUSÃO}

A partir da análise de convergência da solução pela GITT, realizada levando em conta NTP e $N T F$ com $b=0$ e as demais constantes iguais à unidade, conclui-se que nos casos estudados o problema não é influenciado pelo número de termos na solução do problema filtrado, uma vez que quando $b=0$ a solução do problema é uma solução do problema particular. Percebe-se também que a convergência da solução melhora a medida que é aumentado o número de termos da solução particular e que existe uma dificuldade na convergência em $X=1$.

Foi possível observar que os resultados obtidos pela GITT possuem boa concordância com os obtidos pela sub-rotina $F P S 2 H$ para pontos não muito próximos do contorno em $X=1$. Os resultados em $X=1$, em ambos os casos estudados, apresentam comportamento oscilatório, o que é comum em soluções por séries, no entanto, é possível suavizar essa oscilação a medida que se aumenta o número de termos na solução do problema particular. Nota-se também que em $X=1$ esse comportamento é atenuado nos pontos próximos de $Y=1$. 
Conclui-se, deste modo que o objetivo deste trabalho foi alcançado, uma vez que foi demonstrada a complexidade do comportamento do fenômeno de transferência de calor em superfícies estendidas quando a condutividade varia com a temperatura, bem como a técnica empregada foi capaz de resolver os casos propostos alcançando convergências satisfatórias.

\section{REFERÊNCIAS BIBLIOGRÁFICAS}

COTTA, R. M. Hybrid numerical/analytical approach to nonlinear diffusion problems. Num. Heat Transfer, Part B: Fundamentals. v. 17, p. 217-226, 1990;

COTTA, R.M. Integral transforms in computational heat and fluid flow. Boca Raton: CRC Press, 1993;

COTTA, R.M.; MIKHAILOV, M.D. Heat conduction: lumped analysis, Integral transforms, Symbolic Computation. Chichester: John Wiley \& Sons Ltd., 1997;

IMSL Library. Houston: Math/Lib, 1991;

MALIK, M. Y.; RAFIQ, A. Two-dimensional fin with convective base condition. Nonlinear Analysis: Real World Applications. v. 11, p. 147-154, 2010;

RAMOS, R. Análise bidimensional e não-linear de aletas longitudinais de perfil variável via técnica de transformada integral generalizada. Tese (Mestrado em Ciências em Engenharia Mecânica) - Universidade Federal do Rio de Janeiro. Rio de Janeiro, 1993;

SILVA, L. M.; SPHAIER, L. A. Integral transform solution of one-dimensional diffusion problems using an enclosing domain approach. In Proc. of the 6th National Congress of Mechanical Engineering (CONEM). Campina Grande, PB, Brazil, 2010;

SPHAIER, L. A.; COTTA, R. M.; NAVEIRA-COTTA, C. P.; QUARESMA, J. N. N. The UNIT algorithm for solving one-dimensional convection-diffusion problems via integral transforms. I. C. Heat and Mass Transfer. v. 38, p. 565-571, 2011; 